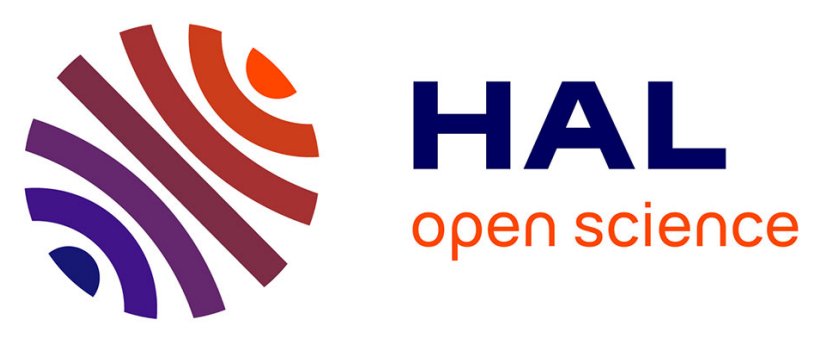

\title{
Characteristics of laser stimulation by near infrared pulses of retinal and vestibular primary neurons
}

Jean-Michel Bec, Emmanuelle S. Albert, Isabelle Marc, Gilles Desmadryl, Cécile Travo, Agnès Muller, Christian Chabbert, Fabrice Bardin, Michel

Dumas

\section{To cite this version:}

Jean-Michel Bec, Emmanuelle S. Albert, Isabelle Marc, Gilles Desmadryl, Cécile Travo, et al.. Characteristics of laser stimulation by near infrared pulses of retinal and vestibular primary neurons. Lasers in Surgery and Medicine, 2012, 44 (9), pp.736-745. 10.1002/lsm.22078 . hal-00744764

\section{HAL Id: hal-00744764 \\ https://hal.science/hal-00744764}

Submitted on 13 Mar 2020

HAL is a multi-disciplinary open access archive for the deposit and dissemination of scientific research documents, whether they are published or not. The documents may come from teaching and research institutions in France or abroad, or from public or private research centers.
L'archive ouverte pluridisciplinaire HAL, est destinée au dépôt et à la diffusion de documents scientifiques de niveau recherche, publiés ou non, émanant des établissements d'enseignement et de recherche français ou étrangers, des laboratoires publics ou privés. 


\title{
Characteristics of Laser Stimulation by Near Infrared Pulses of Retinal and Vestibular Primary Neurons
}

\author{
Jean-Michel Bec, PhD, ${ }^{1}$ Emmanuelle S. Albert, $\mathrm{PhD},{ }^{2}$ Isabelle Marc, $\mathrm{PhD},{ }^{1,3}$ Gilles Desmadryl, PhD, ${ }^{2}$ \\ Cécile Travo, $\mathrm{Ms}^{2}{ }^{2}$ Agnès Muller, $\mathrm{PhD},{ }^{2}$ Christian Chabbert, $\mathrm{PhD},{ }^{2}$ Fabrice Bardin, $\mathrm{PhD},{ }^{1,4 *}$ \\ and Michel Dumas, $\mathbf{P h D}^{1}$ \\ ${ }^{1}$ Institut d'Electronique du Sud, CNRS UMR5214, Université Montpellier 2, Montpellier, France \\ ${ }^{2}$ Institut des Neurosciences de Montpellier, INSERM U-1051, Montpellier, France \\ ${ }^{3}$ Ecole des Mines d'Ales, Nimes, France \\ ${ }^{4}$ Université de Nîmes, Nîmes, France
}

Background and Objective: The optical stimulation of neurons from pulsed infrared lasers has appeared over the last years as an alternative to classical electric stimulations based on conventional electrodes. Laser stimulation could provide a better spatial selectivity allowing single-cell stimulation without prerequisite contact. In this work we present relevant physical characteristics of a non-lethal stimulation of cultured mouse vestibular and retinal ganglion neurons by single infrared laser pulses. Study Design/Materials and Methods: Vestibular and retinal ganglion neurons were stimulated by a 100-400 $\mathrm{mW}$ pulsed laser diode beam (wavelengths at 1,470, 1,535, $1,875 \mathrm{~nm}$ ) launched into a multimode optical fiber positioned at a few hundred micrometers away from the neurons. Ionic exchange measurements at the neuron membrane were achieved by whole-cell patch-clamp recordings. Stimulation and damage thresholds, duration and repetition rate of stimulation and temperature were investigated.

Results: All three lasers induced safe and reproducible action potentials (APs) on both types of neurons. The radiant exposure thresholds required to elicit APs range from $15 \pm 5$ to $100 \pm 5 \mathrm{~J} \mathrm{~cm}^{-2}$ depending on the laser power and on the pulse duration. The damage thresholds, observed by a vital dye, were significantly greater than the stimulation thresholds. In the pulse duration range of our study (2-30 milliseconds), similar effects were observed for the three lasers. Measurements of the local temperature of the neuron area show that radiant expo-sures required for reliable stimulations at various pulse durations or laser powers correspond to a temperature in-crease from $22^{\circ} \mathrm{C}$ (room temperature) to $55-60^{\circ} \mathrm{C}$. Stimu-lations by laser pulses at repetition rate of 1,2 , and $10 \mathrm{~Hz}$ during 10 minutes confirmed that the neurons were not damaged and were able to survive such temperatures. Conclusion: These results show that infrared laser radi-ations provide a possible way to safely stimulate retinal and vestibular ganglion neurons. A similar temperature threshold is required to trigger neurons independently of variable energy thresholds, suggesting that an absolute temperature is required.
Key words: laser; retinal ganglion neuron; vestibular ganglion neuron

\section{INTRODUCTION}

Classical neuronal stimulation techniques (such as electrical or pharmacological) suffer from spatial and temporal limitations and can result in possible mechanical damages to the tissue. Optical stimulation methods of neurons have been recently developed which could overcome these drawbacks [1]. Various strategies have been reported to optically stimulate neurons which are not normally photo-activated, including optical release of caged glutamate from UV irradiation $[1,2]$ or genetically adapted light-activated ionic channels [3-10]. Genetic integration of cation channels such as channelrhodopsin-2 or halorhodopsin has allowed cell stimulation with blue or yellow light $[4,5]$. Possible applications include reactivation of light-insensitive human photoreceptors in diseases such as retinitis pigmentosa [9] or technology for probing mammalian brain structures [10].

A similar optical stimulation method based on infrared light, appearing as an effective contactless method to evoke neural activity in motor or sensory systems and requiring no cell property modifications is also currently investigated [11-23]. The multiphoton excitation from a femtosecond Ti:sapphire laser light on neurons from neocortical brain slices was demonstrated in 2002 [11]. Later, in vivo rat and leopard frog sciatic nerve stimulations

Conflict of Interest Disclosures: All authors have completed and submitted the ICMJE Form for Disclosure of Potential Conflicts of Interest and none were reported.

Fabrice Bardin and Michel Dumas contributed equally to this work.

Contract grant sponsor: European Aeronautic Defence and Space Corporate Foundation; Contract grant sponsor: FAF (Fédération des Aveugles et Handicapés Visuels de France); Contract grant sponsor: UNADEV (Union Nationale des Aveugles et Déficients Visuels).

*Corresponding to: Fabrice Bardin, $\mathrm{PhD}$, Université de Nîmes, Site des Carmes, Place Gabriel Péri, 30000, Nîmes, France.

E-mail: fabrice.bardin@unimes.fr 
were also reported using microsecond to millisecond pulses from near infrared lasers (wavelengths at 1,870, $2,100-6,100 \mathrm{~nm})[12,13]$. Similar results were obtained on in vivo cochlear neurons of gerbils using microsecond pulses of a diode laser with a wavelength of $1,940 \mathrm{~nm}$ [14-16], on rat sciatic nerves with a $1,500 \mathrm{~nm}$ laser source [17], and more recently on rat cavernous nerves [18] and on balance organs of the inner ear [19]. The IR laser stimulation of cultured cells was also demonstrated, on isolated rat hippocampal neurons irradiated by a $980 \mathrm{~nm}$ laser [20], on transfected HEK293 cells by a 1,460 nm laser [21], on neonatal cardiomyocyte cells by a 1,862 $\mathrm{nm}$ laser [22] and on oocytes, untransfected HEK cells and artificial lipid bilayers [23]. These studies have demonstrated the safety of nerve tissue stimulation by infrared light source without the need of any tissue property modification. They have also defined an optimum wavelength range between 1,000 and $2,000 \mathrm{~nm}$ by establishing a relationship with the absorption coefficient of water [12]. These authors suggest that among the main biophysical mechanisms underlying the laser-tissue interaction (photochemical, photothermal, photomechanical, photoablation) [24], the photothermal process is the most likely mechanism, with an estimated temperature rise of $0.1^{\circ} \mathrm{C}$ [16], of $4-6^{\circ} \mathrm{C}$ [13], of $14^{\circ} \mathrm{C}$ [20], of $22^{\circ} \mathrm{C}$ [23], or of $35^{\circ} \mathrm{C}$ [21]. This heating has been shown to reversibly change the electrical capacitance of the plasma membrane of oocytes, HEK cells and artificial bilayers, depolarizing the target cell [23]. These results are consistent with the mechanism presented in the literature [24] occurring at such a light energy delivered per unit area (from $0.5 \mathrm{~J} / \mathrm{cm}^{2}$ to few tens of $\mathrm{J} / \mathrm{cm}^{2}$ ) and exposure duration (in a millisecond regime).

In this article, we report on the infrared laser stimulation characteristics on two different types of cultured sensory primary neurons: rat vestibular Scarpa's ganglion and mouse retinal ganglion neurons. The laser irradiation characteristics inducing safe neuron biological responses were investigated from electrophysiological recordings (whole cell patch-clamp method). We show that a small range of energy operated from three fiber pigtailed laser diodes (wavelengths at 1,470, 1,535, and 1,875 nm) successfully activates action potentials (APs), depending on the pulse duration, power and wavelength of the laser and on the positioning of the optical fiber tip. By measuring the temperature rise at the optical fiber output, we also show that the stimulation thresholds correspond to a constant temperature rise, supporting the hypothesis of a photothermal stimulation.

\section{MATERIALS AND METHODS}

\section{Culture of Ganglion Neurons}

All the following experiments were conducted in vitro using vestibular ganglion neurons (VGNs) cultured from rats and retina ganglion neurons (RGNs) isolated from C57BL/6J mice and were carried out in accordance with the European Communities Council Directive 86/609/ EEC.
VGNs were aseptically explanted from 3-day-old Wistar rats (Centre d'Elevage Janvier, Le Genest-Saint-Isle, France). The brainstem was hemi-sectioned and the eighth nerve was followed up to the level of the otocyst and cut just rostral to it. The vestibular ganglia were excised, placed in Leibovitz medium (L15, Invitrogen, Cergy Pontoise, France), and enzymatically dissociated (collagenase $0.75 \mathrm{mg} / \mathrm{ml}$, dispase $1 \mathrm{mg} / \mathrm{ml}$, and DNase $0.75 \mathrm{mg} / \mathrm{ml}, 15$ minutes at $25^{\circ} \mathrm{C}$ ). Enzymes were washed with Tyrode solution without $\mathrm{Ca}^{2+}$ and $\mathrm{Mg}^{2+}$, and ganglion neurons were gently dissociated in culture medium with a pipette. Neurons were then plated onto glass bottom culture dishes previously coated with $5 \mu \mathrm{g} / \mathrm{ml}$ poly-L-ornithine, $10 \mu \mathrm{g} / \mathrm{ml}$ laminin and subsequently filled with $0.5 \mathrm{ml}$ of a 1:1 mixture of Dulbecco's modified Eagle's medium and Ham's F-12 medium supplemented with $2 \% \mathrm{~N}_{2}$ nutrient glucose $(5 \mathrm{~g} / \mathrm{L})$, glutamine $(1.5 \mathrm{mM})$, sodium bicarbonate $(1.1 \mathrm{~g} / \mathrm{L})$, HEPES buffer $(15 \mathrm{mM}$, $\mathrm{pH} 7.4)$, AraC $(2 \mu \mathrm{M}), 10 \mu \mathrm{g} / \mathrm{ml}$ brain-derived neurotropic factor and $1 \%$ penicillin/streptomycin. Low density cultures were then maintained at $37^{\circ} \mathrm{C}$ in $5 \% \mathrm{CO}_{2}$.

Postnatal (P2) C57BL/6J mice (Janvier Institute, Le Genest-St-Isle, France) were decapitated. Retinas were removed and transferred in warm $\left(37^{\circ} \mathrm{C}\right)$ phosphatebuffered saline (PBS) medium and then enzymatically dissociated in PBS containing papain $(33 \mathrm{U} / \mathrm{ml}$, Worthington Biochemical Corporation, Lakewood, NJ) for 30 minutes at $37^{\circ} \mathrm{C}$. They were then mechanically and gently triturated in PBS containing $1 \%$ ovomucoid (Worthington Biochemical Corporation) and DNase (100 U/ml). Dissociated RGNs were incubated for 15 minutes at $4{ }^{\circ} \mathrm{C}$ with mouse anti-mouse CD90 Thy1.2 antibodies conjugated to microbeads (Macs Cells Separation Columns, Miltenyi Biotech, Paris, France) in PBS with $0.2 \%$ bovine serum albumin (BSA). After washing, neurons were eluted using a plunger. Approximately 20,000 purified RGNs per $\mathrm{cm}^{2}$ were cultured onto glass bottom dishes coated with poly-D-lysine $(20 \mu \mathrm{g} / \mathrm{ml}$ for 2 hours at $\left.37^{\circ} \mathrm{C}\right)$. Finally, neurons were incubated in supplemented neurobasal at $37^{\circ} \mathrm{C}$ in a $5 \% \mathrm{CO}_{2}$ humidified atmosphere.

\section{Electrophysiological Recordings}

The whole-cell patch-clamp recordings were performed in current clamp mode on an inverted microscope (Nikon Instruments Europe B.V., Amstelveen, The Netherlands). Currents were recorded and amplified using an Axopatch 200B amplifier (Axon Instruments, Downingtown, PA), Digidata 1440 board and pClamp 10 software (Molecular Devices, Sunnyvale, CA). Signals were also synchronized with the laser stimulation with a multifunctional data acquisition card (NI PCI 6221, National Instruments, Austin, TX) and a homemade National Instrument's LabVIEW program (National Instruments). The pipette series resistance and capacitance were compensated using the built-in circuitry of the amplifier, and the liquid junction potential between the pipette and the bath was zeroed before seal formation. The pipettes were fabricated from borosilicate glass (Sutter Instruments, Novato, CA) 
with an initial access resistance less than $6 \mathrm{M} \Omega$. All voltages were defined as membrane potentials with respect to extracellular solutions.

The viability of neurons was checked by using a vital dye in order to measure the damage thresholds. Neurons were exposed to $0.5 \mathrm{ml}$ of $0.4 \%$ Trypan Blue (Invitrogen), added to the extracellular medium (2.5 ml) [25]. Neurons were then irradiated by laser pulses delivered at repetition rate of one $\mathrm{Hz}$ during 1 minute and observed with a camera (NET FOculus, Model FO124B, Finning, Germany) during 5 minutes before another irradiation. Pharmacological tests included tetrodotoxine (TTX, $1 \mu \mathrm{M}$, from Sigma Aldrich, Lyon, France) added to the bathing medium in the vicinity of the neuron by puff or by using a fast gravity perfusion system.

\section{Laser Stimulation Set-Up}

Figure 1 provides a schematic view of the experimental set-up. Three pigtailed laser diodes (Sheaumann Laser, Inc., Marlborough, MA) emitting in the near infrared at $1,875,1,535$, and $1,470 \mathrm{~nm}$ were used (fiber type: 105/125, $0.22 \mathrm{NA}$ ). They were mounted on an air-cooling block and operated at room temperature. A multimode fiber of $105 \mu \mathrm{m}$ core diameter (0.22 NA) (Thorlabs, Inc., Newton, $\mathrm{NJ})$ was cleaved and polished to expose the fiber core on one end while its other end was connected via an SMA connector to the fiber output of the laser diodes. The optical fiber was mounted on an $x-y-z$ translator attached to a micromanipulator (Narishige, Tokyo, Japan). A visible light source was also coupled to the fiber for alignment to the target area, before switching to the infrared laser.

All the laser stimulation parameters (radiant exposure, pulse duration, repetition rate) were totally controlled through a LabVIEW program. This program controls the laser diode driver (ILX Ligthwave, Bozeman, MT, Model LDX 3232) by a computer data acquisition and control board (National Instruments, Model NI PCI 6221). Moreover, it provides the recording of signals at a sampling rate of $25 \mathrm{kHz}$ and the generation of a single pulse with a duration as short as 10 microseconds at the driver maximum output current of $4 \mathrm{~A}$. At the bare fiber output, we measured a maximum output power (obtained at $3.5 \mathrm{~A}$ ) of $230 \mathrm{~mW}$ for the $1,875 \mathrm{~nm}$ laser, $410 \mathrm{~mW}$ for the $1,535 \mathrm{~nm}$ laser and $430 \mathrm{~mW}$ for the $1,470 \mathrm{~nm}$ laser with a powermeter (Coherent, Courtabœuf, France, Model Fieldmate).

\section{Temperature Measurement at Cellular Level}

Classical methods of temperature detections are not suitable for cell temperature measurements. For instance, electrical temperature sensors (as thermocouple or PT100) have insufficient accuracy and behave as a black body under IR wavelength. Thermal cameras can measure the surface temperature of the bath or a tissue [13], which significantly differs from the temperature of the neuron placed a few millimeters below the bath surface [26].

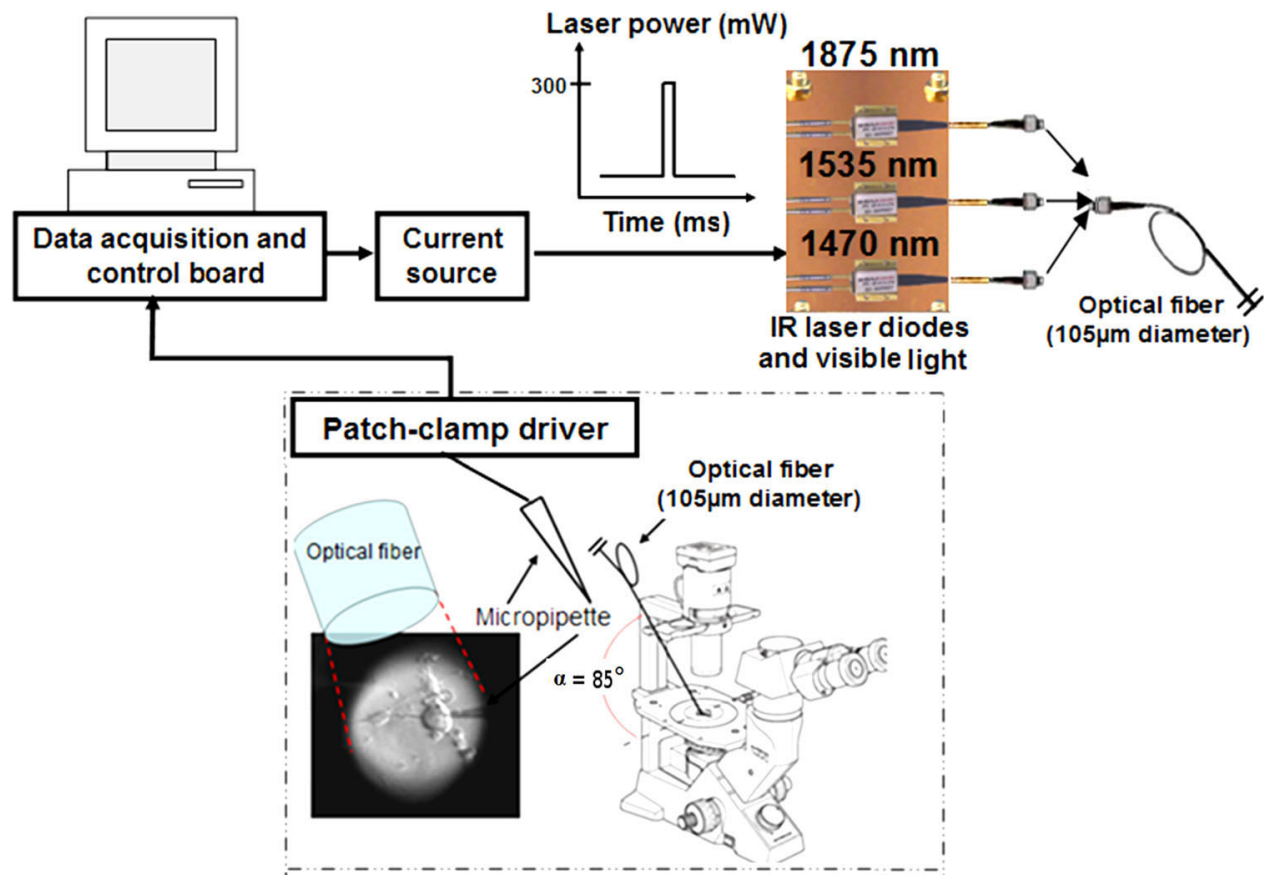

Fig. 1. Schematic illustration of the experimental setup including the optical stimulation of neurons from infrared laser diode light observed by electrophysiological recordings (by whole cell path clamp method). The IR beam is guided through an optical fiber with a core diameter of $105 \mu \mathrm{m}$. The fiber tip was positioned $165 \mu \mathrm{m}$ from the neuron. The pigtailed laser diodes were powered by the modulation of a computer controlled continuous current source. [Color figure can be seen in the online version of this article, available at http:// wileyonlinelibrary.com/journal/lsm] 


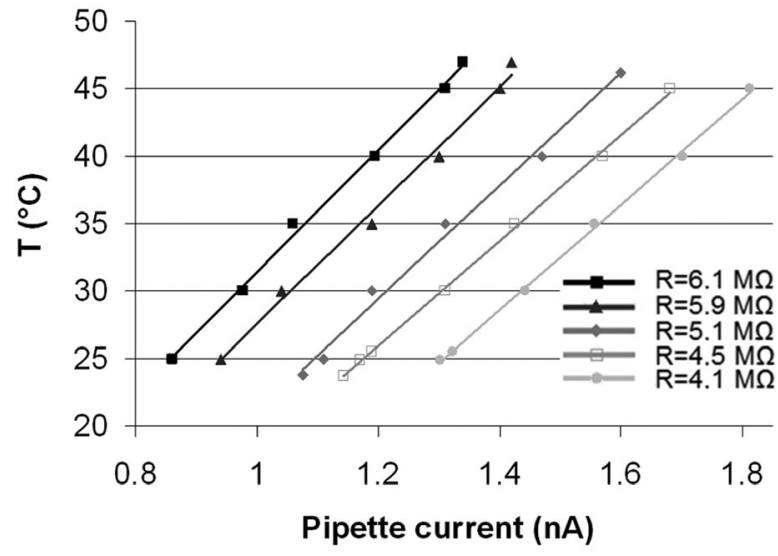

Fig. 2. Experimental calibration of the pipette current function of the temperature of the bath for various values of the initial pipette conductance. The chamber solution was preheated to $45^{\circ} \mathrm{C}$ and then passively cooled. The pipette current (induced by a square voltage command pulse of $5 \mathrm{mV}$ ) and the temperature of the solution were simultaneously recorded.

Several authors have shown that local temperatures could be monitored by irradiating the tip of an open patch pipette [21,23,27-29]. The local temperature change induces a resistance variation of the electrode tip. Calibrations were performed with the bath solution which was preheated beforehand (around $45-50^{\circ} \mathrm{C}$ ) and passively cooled down to $24^{\circ} \mathrm{C}$. The bath temperature was simultaneously monitored by using an immersed thermometer. A square voltage command pulse of $5 \mathrm{mV}$ was applied to generate a current through the open pipette. Figure 2 illustrates the quasi-linear evolution of current as a function of the temperature in the range from 25 to $50^{\circ} \mathrm{C}$ for different values of the initial pipette resistance. Such a behavior has been previously described [29].

\section{RESULTS}

Reproducible and non-lethal APs were validated from stimulations of all three lasers ( $n=18 \mathrm{RGNs}, n=43$ VGNs). An example of APs stimulated by 4 milliseconds single laser pulses is shown in Figure 3. The laser evoked membrane voltage variations usually displayed an initial hump-like depolarization (mean maximum amplitude: $10 \pm 4 \mathrm{mV}, n=12 \mathrm{RGNs}$ and $10 \pm 5 \mathrm{mV}, n=25 \mathrm{VGNs}$ ), on top of which a spike-like component (mean peak amplitude: $30 \pm 5 \mathrm{mV} ; n=12 \mathrm{RGNs}$ and $35 \mathrm{mV} \pm 5 \mathrm{mV}$; $n=25 \mathrm{VGNs}$ ) occurred. The hump-like depolarization phase always started within the first millisecond after the onset of the irradiation. Conversely, the occurrence of the spike component could be delayed up to 10 milliseconds under our irradiation conditions.

\section{Energy Received by the Neurons}

A simulation program was developed to assess the accurate energy received by the neurons. A Matlab (Mathworks, Natick, MA) algorithm allowed the calculation

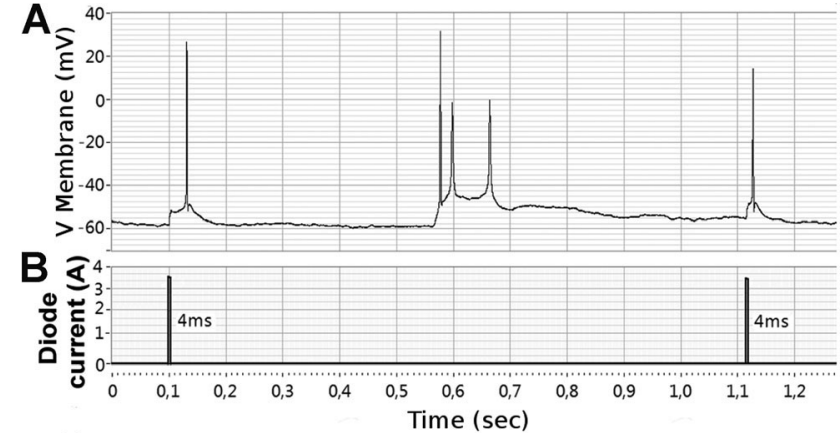

Fig. 3. A: Action potentials on a VGN stimulated by 4 milliseconds single laser pulses at $1,875 \mathrm{~nm}$. B: Laser diode current pulses synchronized with the membrane potential recording. Three spontaneous APs were also observed between two stimulations.

of the radiant exposure received by the neurons at any position in the laser spot by taking into account the Gaussian characteristic of the laser beam at the optical fiber output. It also takes into account the specificities of the measurement methods: fiber insertion angle, neuron position in the laser spot, distance between the fiber and the neurons, water absorption coefficient for each wavelength.

In the mid infrared wavelength range, the absorption coefficient of water is relatively high and varies rapidly. The absorption coefficient of water for a beam at 1,875 or $1,470 \mathrm{~nm}$ has a similar value around $30 \mathrm{~cm}^{-1}$ whereas it is $13 \mathrm{~cm}^{-1}$ at $1,535 \mathrm{~nm}$ [30]. The algorithm considers the radiant power decrease proportionally to the distance between the fiber tip and the neurons. As described in Figure 4 , the insertion angle of the fiber is around $85^{\circ}$ since the inverted microscope configuration allows free space, leading to a nearly symmetrical Gaussian spot (Fig. 4B). The fiber tip was located vertically at $165 \pm 10 \mu \mathrm{m}$ ( $Z$-axis) above the irradiated neurons. The center of the neuron soma was also positioned in the range $40-55 \mu \mathrm{m}$ from the spot center as illustrated on the neuron photograph in Figure 1.

Finally, the energy per unit area (radiant exposure) received by both types of neurons was calculated by taking into account the position of the light beam and the nonuniformity of the laser beam on the neuron surface (with a soma diameter of around $20 \mu \mathrm{m}$ for RGNs and around $40 \mu \mathrm{m}$ for VGNs). As described in Figure 4B, the lateral position of the beam can induce a significant variation of radiant exposure received by the neurons, in particular when the neuron is positioned on the periphery of the Gaussian peak center.

\section{Stimulation Thresholds}

We have successfully stimulated both RGNs and VGNs with an optical radiation at a wavelength of $1,875 \mathrm{~nm}$ over a large range of powers and single pulse durations. To estimate the stimulation thresholds, the pulse duration was increased by steps at constant power. Figure 5A illustrates the mean and the standard deviation of the 

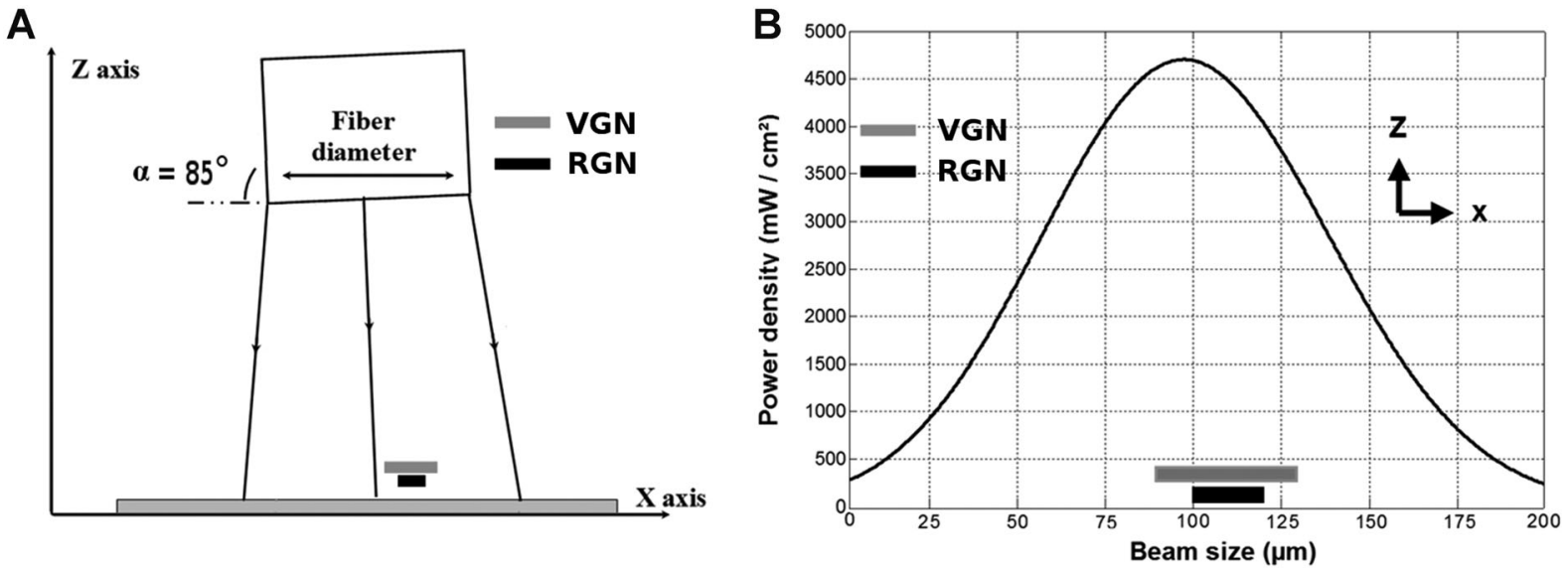

Fig. 4. A: Position of the optical fiber with an insertion angle of $85 \pm 4^{\circ}$. B: Gaussian distribution of the radiant power at the neuron surface.

pulse duration required to stimulate RGNs or VGNs for different values of radiant power at the output of the bare end fiber $(n=11$ for VGNs and $n=8$ for VGNs at $230 \mathrm{~mW} ; n=3$ for RGNs and for VGNs for other radiant powers and pulse durations). RGNs and VGNs stimulation threshold parameters (mean and standard deviation) have been found similar. For the sake of clarity, all RGNs results are not shown in Figure 5. A similar general trend is obtained whatever the neuron type. A decrease of the radiant power requires an increase of the pulse duration. Conversely, the radiant exposure increases as the pulse duration gets larger.

Addition of tetrodotoxin, a specific blocker of the voltage-gated sodium channels [31], reversibly abolished the spike-like component of the laser evoked biological response in the two neuron types $(n=4 \mathrm{RGNs}$ and $n=7$ VGNs) as shown by Albert et al. [32]. This effect identifies the spike-like component as a sodium-based AP.

\section{Wavelength Effect on Radiant Exposure Thresholds}

We have also studied the radiant exposure threshold variation as a function of the wavelength. We have successfully stimulated RGNs and VGNs with a laser beam at $1,470,1,535$, and $1,875 \mathrm{~nm}$ for various pulse durations and powers. As previously described at $1,875 \mathrm{~nm}$, both neuron types follow a similar stimulation threshold trend. For the sake of clarity, only the mean stimulation thresholds (and the standard deviation) measured on VGNs are displayed in Figure 6. The radiant power at the fiber output function of the pulse duration is plotted for the three wavelengths in Figure 6A. The radiant exposure thresholds received by the neurons, deduced from the mean radiant power thresholds, are shown in Figure 6B. At 1,470 and at $1,535 \mathrm{~nm}$, we can again observe that the radiant exposure threshold decreases as the exposure duration decreases.

\section{Damage Thresholds}

The viability of the neurons was checked by exposing no-patched neurons to a vital dye (Trypan Blue). The mean damage thresholds were determined when neurons were not able to exclude the stain while increasing the pulse duration by step of 1 millisecond at constant radiant power ( $n=8$ at $230 \mathrm{~mW}, n=3$ for the other powers). Gray symbols in Figure 5 and in Figure 6 illustrate the mean and the standard deviation of the damage thresholds for different laser powers. These results clearly show that Trypan Blue did not stain neurons irradiated in the stimulation domain.

The neuron staining was also accompanied by a neuron swelling. In addition, a loss of stabilized resting potential after spikes in the patch-clamp configuration was observed at and upper the damage thresholds. Bubble formation occurred with a pulse duration of 80 milliseconds at $230 \mathrm{~J} / \mathrm{cm}^{2}$ with the $1,875 \mathrm{~nm}$ laser. Vogel and Venugopalan [33] have shown that bubble formation in water could be induced at $1,590 \mathrm{~J} / \mathrm{cm}^{2}$ with a $2,000 \mathrm{~nm}$ laser (with a pulse duration of 0.2 milliseconds) and at $80 \mathrm{~J} / \mathrm{cm}^{2}$ with a $2,100 \mathrm{~nm}$ laser (pulse duration of 0.2 milliseconds).

\section{Stimulated APs at 1, 2, and $10 \mathrm{~Hz}$}

In order to check the viability of the neurons under realistic stimulation parameters, the neurons were stimulated by laser pulses at repetition rate of 1,2 , and $10 \mathrm{~Hz}$ during 10 minutes each $(n=2)$. Figure 7 shows stimulated APs in these configurations. A constant radiant exposure was able to elicit APs for more than half an hour. No decrease of the spike amplitude and no neuron adaptation were observed.

\section{Temperature Measurements}

Figure $8 \mathrm{~A}$ outlines an example of the pipette currents measured in the open patch pipette configuration and their associated temperature rises determined from calibration data in Figure 2, for single pulse durations of 7, 10 , and 20 milliseconds. Thermal diffusion is clearly seen for a pulse duration of 20 milliseconds.

We investigated the spatial variation of temperature within the beam by radially moving the tip of the open 

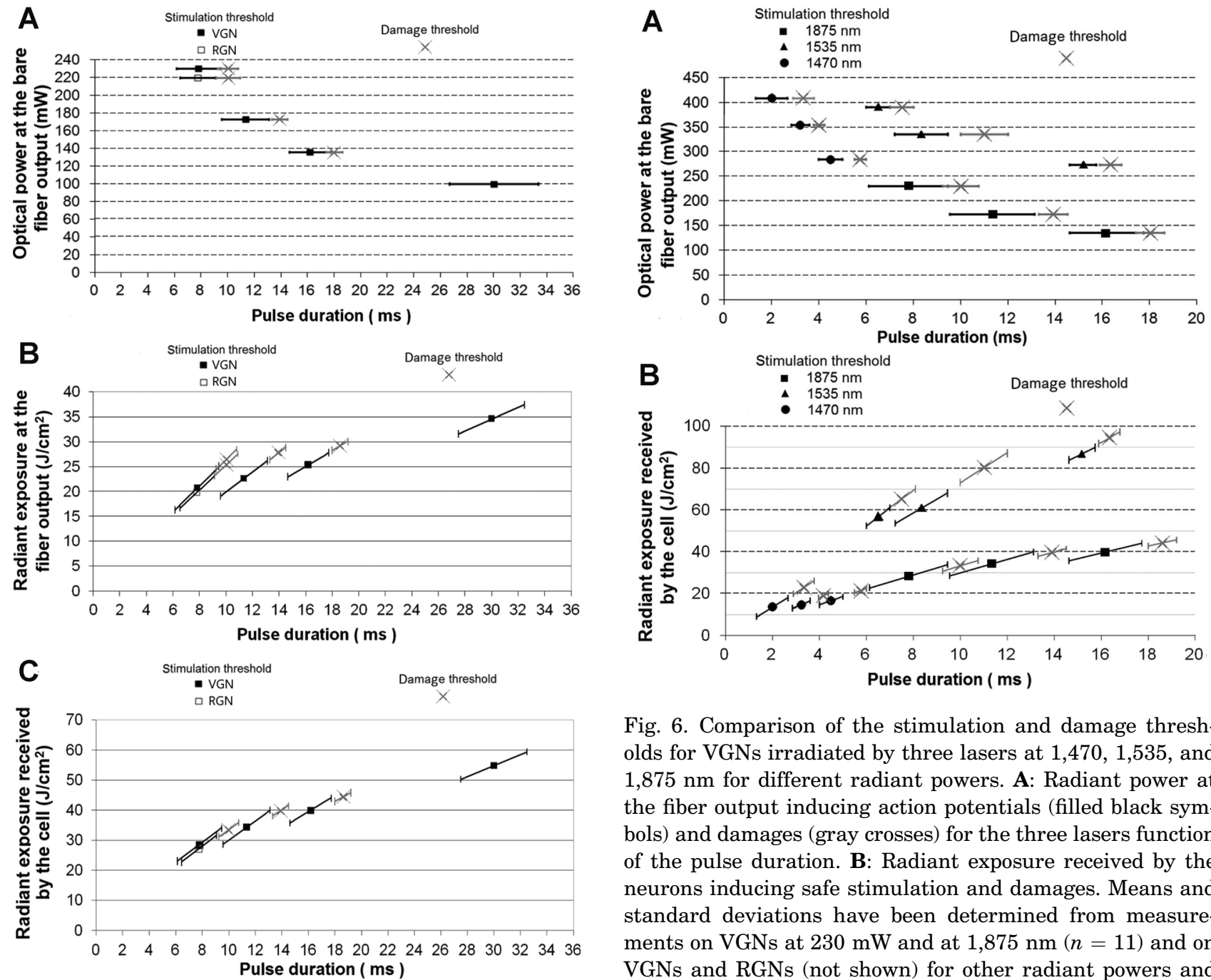

Fig. 6. Comparison of the stimulation and damage thresholds for VGNs irradiated by three lasers at 1,470, 1,535, and $1,875 \mathrm{~nm}$ for different radiant powers. A: Radiant power at the fiber output inducing action potentials (filled black symbols) and damages (gray crosses) for the three lasers function of the pulse duration. B: Radiant exposure received by the neurons inducing safe stimulation and damages. Means and standard deviations have been determined from measurements on VGNs at $230 \mathrm{~mW}$ and at $1,875 \mathrm{~nm}(n=11)$ and on VGNs and RGNs (not shown) for other radiant powers and laser wavelengths $(n=3)$.

Fig. 5. Stimulation and damage thresholds of RGNs and VGNs irradiated by a single pulse of a laser beam at $1,875 \mathrm{~nm}$ for different radiant powers. A: Radiant power at the output of the bare fiber required to induce APs (filled black symbols) and damages (gray crosses) observed by a vital dye. Stimulation and damage radiant exposure $(\mathbf{B})$ at the fiber output and (C) at the neuron surface. Means and standard deviations have been determined from measurements on VGNs $(n=11)$ and on RGNs $(n=8)$ at $230 \mathrm{~mW}$ and on RGNs and VGNs for other radiant powers and pulse durations $(n=3)$. For the sake of clarity, RGNs signals (open symbol) were plotted at $220 \mathrm{~mW}$ whereas the neurons were tested at $230 \mathrm{~mW}$.

pipette from the beam center to its periphery. In a position similar to the one with a neuron (165 $\mu \mathrm{m}$ above the pipette tip), an almost circular spot of $220 \mu \mathrm{m}$ diameter was generated. Figure $8 \mathrm{~B}$ exhibits the peak temperature function of the central position obtained while irradiating the tip within the optimum radiant exposure threshold condition (i.e., the smallest pulse duration) for the three

wavelengths. This corresponds to a single pulse duration of 2 milliseconds at 1,470 nm, 6 milliseconds at 1,535 nm and 7 at $1,875 \mathrm{~nm}$. A temperature decrease of $30 \%$ is observed from the center to the periphery of the spot. Neurons were irradiated between the center and the periphery of the visible spot (radial position between 36 and $74 \mu \mathrm{m}$ ) due to spatial crowding of the pipette.

A similar study was carried out to measure the vertical spatial variation of the temperature at the fiber output. The pipette tip was moved vertically from 130 to $40 \mu \mathrm{m}$ to the fiber tip while measuring the temperature for the three wavelengths at a constant radial position of $55 \mu \mathrm{m}$. As expected, a temperature decrease was observed while moving away the fiber tip (Fig. 8C).

Figure 8D shows the temperature rise for various pulse durations at maximum and constant radiant power for the three wavelengths (at a constant radial position of $55 \mu \mathrm{m})$. The results show identical temperature variations at $1,875 \mathrm{~nm}$ and at $1,535 \mathrm{~nm}$ whereas at $1,470 \mathrm{~nm}$ higher temperatures are reached. Finally, we examined 


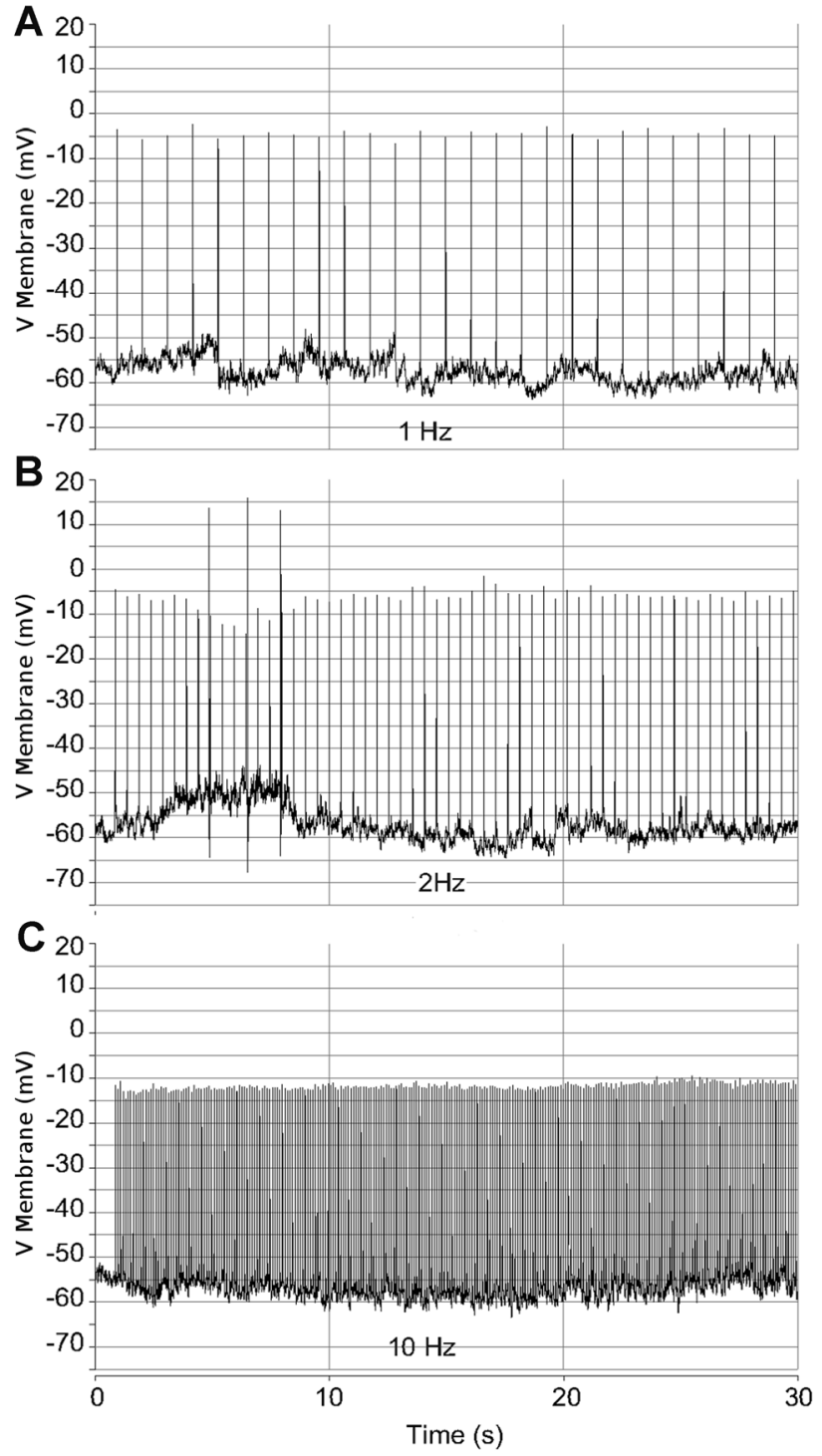

Fig. 7. Membrane potential variations under irradiation of laser pulses at repetition rate of $(\mathbf{A}) 1 \mathrm{~Hz},(\mathbf{B}) 2 \mathrm{~Hz}$, and $(\mathbf{C})$ $10 \mathrm{~Hz}$ with a pulse duration of 8 milliseconds (i.e., $31 \mathrm{~J} / \mathrm{cm}^{2}$ ). The laser wavelength is $1,875 \mathrm{~nm}$. Each repetition rate was applied during 10 minutes $(n=2)$. The traces are 30 seconds long for clarity.

in Figure 8E the temperature rise for every radiant exposure thresholds previously presented in Figure 6B.

\section{DISCUSSION}

We have successfully demonstrated safe and reproducible vestibular and retinal ganglion neuron laser stimulation using single pulses from near infrared lasers from whole cell path clamp recordings.

Because of the high absorption of near or mid infrared wavelengths in water, the energy received by neurons depends on the position of the optical fiber tip. We have calculated the radiant exposure received by the neurons by taking into account not only the position of the fiber tip but the neuron size and the Gaussian beam characteristics. Energy thresholds at the cell level have the same magnitude for both neuron types. Stimulation thresholds also follow the same trend as a function of the pulse duration. This suggests that a similar mechanism takes place for both neuron types. Our approach gives threshold values of radiant exposure $\left(20-100 \mathrm{~J} / \mathrm{cm}^{2}\right)$ greater than those reported in in vivo studies on the stimulation of auditory system of gerbils $\left(0.018-0.003 \mathrm{~J} / \mathrm{cm}^{2}\right)$ [14] and on the rat sciatic nerve $\left(0.3-0.4 \mathrm{~J} / \mathrm{cm}^{2}\right)[13],\left(3.7 \mathrm{~J} / \mathrm{cm}^{2}\right)$ [17] but in the same energy range as laser stimulation of cultured neurons in almost similar conditions $\left(10 \mathrm{~J} / \mathrm{cm}^{2}\right.$ with a pulse duration of 4 milliseconds on neonatal cardiomyocyte cells [22], $3.7 \mathrm{~mJ}$ with a pulse duration of 1 millisecond on HEK cells [23]).

The radiant exposure required to elicit APs increases while increasing the pulse duration. Several authors have already reported similar results $[13,16]$. The laser energy is strongly absorbed by the water molecules and converted to heat which is localized closely to the fiber tip. Due to the diffusion phenomenon, the heat is no longer completely confined for long pulses. As described by Izzo et al. [16], a constant radiant exposure threshold is found with pulses below 100 microseconds. Since the lasers and the current source we used were limited in power, we were not able to stimulate neurons below 2 milliseconds.

Again, the absorption coefficient has a major role on the stimulation threshold. Our lasers at $1,535 \mathrm{~nm}$ and at $1,470 \mathrm{~nm}$ have the same maximum radiant power (around $400 \mathrm{~mW}$ ) but the radiant exposure threshold is clearly higher at $1,535 \mathrm{~nm}$ than at $1,470 \mathrm{~nm}$. This can be explained by the difference of their water absorption coefficient: $15 \mathrm{~cm}^{-1}$ at $1,535 \mathrm{~nm}$ and $30 \mathrm{~cm}^{-1}$ at $1,470 \mathrm{~nm}$. As the beam is less absorbed at $1,535 \mathrm{~nm}$, it requires more energy to trigger the neuron. We can observe the same effect by comparing thresholds and pulse durations at 1,875 and $1,470 \mathrm{~nm}$. For the latter two wavelengths, the absorption coefficient is similar $\left(30 \mathrm{~cm}^{-1}\right)$ but the laser at $1,875 \mathrm{~nm}$ has almost half the maximum power of the laser at $1,470 \mathrm{~nm}$. The radiant exposure thresholds of both lasers clearly follow the same trend. We would expect to obtain superposed thresholds if the $1,875 \mathrm{~nm}$ laser was more powerful.

All three lasers were able to elicit non-lethal APs. No staining of a vital dye was observed on neurons irradiated at the radiant exposure thresholds. A very large majority of the damage thresholds are clearly out of the stimulation thresholds. All three lasers were proved to be equally effective since no significant variations were found between both threshold domains whatever the laser wavelength. In the power and the pulse duration ranges of our study, we found no significant effect on the gap between both threshold domains while varying the laser power. Shortest pulse durations are used for the 1,470 nm laser. This could allow maximizing the repetition rate. Future work includes investigating the effect of shorter pulse duration by using a 10 A current source. 


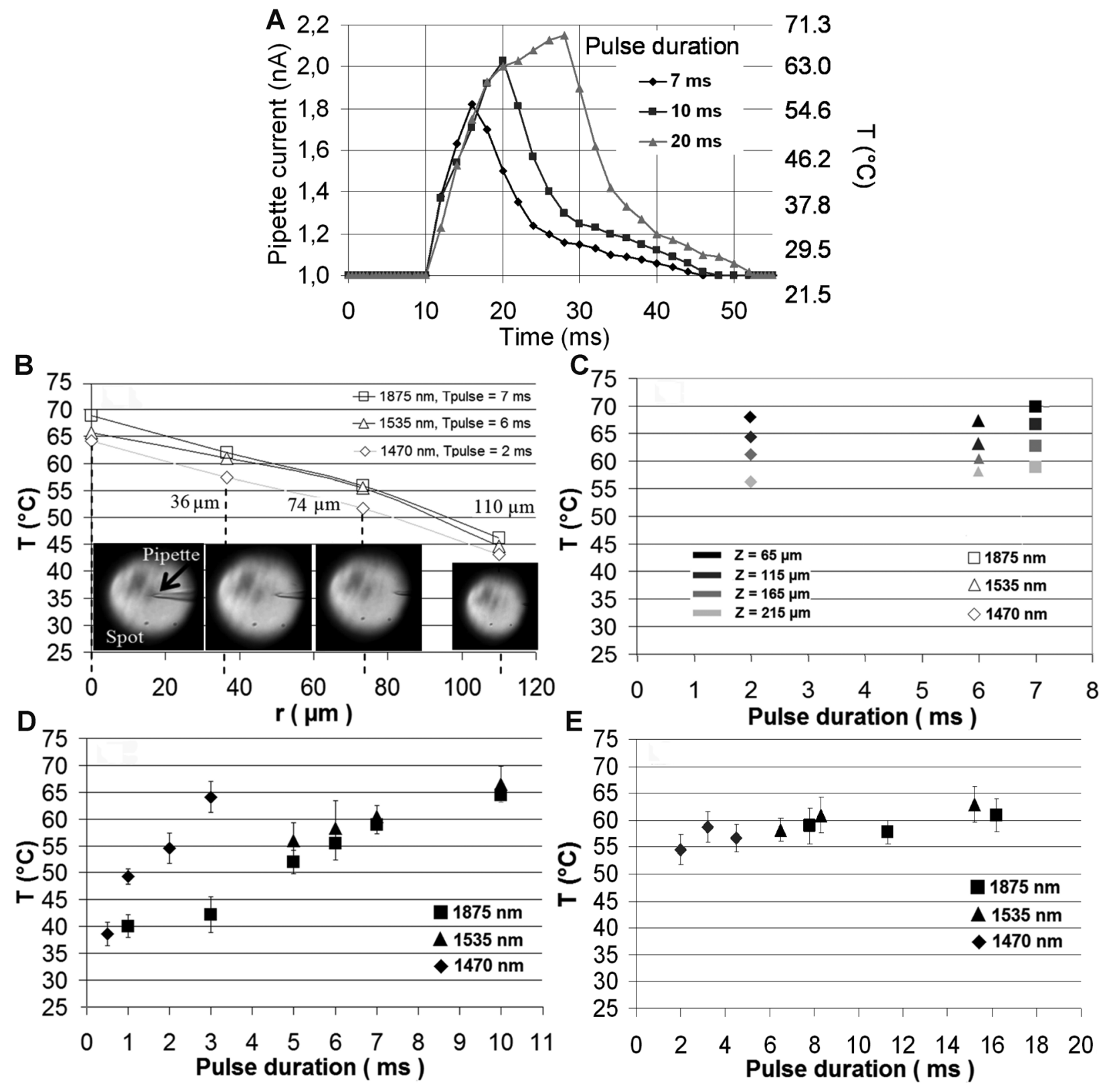

Fig. 8. Temperature measurements during laser irradiation of a saline bath. A: Pipette currents measured in the open patch pipette configuration and their associated temperature rises determined from calibration data in Figure 2, for single pulse durations of 7, 10, and 20 milliseconds with the $1,875 \mathrm{~nm}$ laser. B: Radial spatial variation of the temperature under irradiation of the beam at the maximum radiant power and at the smallest pulse duration evoking an AP. This corresponds to a pulse duration of 2 milliseconds at 1,470 nm, 6 milliseconds at $1,535 \mathrm{~nm}$ and 7 at $1,875 \mathrm{~nm}$. The pipette tip was positioned as in a normal patch clamp recording test ( $165 \mu \mathrm{m}$ below the fiber tip). C: Vertical spatial variations of the temperatures for various pulse durations. The open pipette tip was vertically moved from 65 to $215 \mu \mathrm{m}$ below the fiber tip at a constant radial position of $55 \mu \mathrm{m}$ from the center of the spot. D: Temperature rise for various pulse durations at maximum and constant radiant power for the three wavelengths at a radial position of $55 \mu \mathrm{m}$ from the center of the spot and at $165 \mu \mathrm{m}$ below the fiber tip. E: Temperature rise for every radiant exposure thresholds presented in Figure 6B. 
The likely mechanism by which the biological response to the optical stimulation occurs is the increase of temperature on neurons upon light absorption by water. The measurements of the local temperature of the neuron area by the open pipette technique show that radiant exposures able to elicit APs at various wavelengths and various pulse durations or laser powers correspond to a temperature rise from $22^{\circ} \mathrm{C}$ (room temperature) to 55$60^{\circ} \mathrm{C}$. It is interesting to note that a same temperature threshold is obtained for various stimulation conditions, suggesting that an absolute temperature is the key parameter more than the rate of heat. This result is consistent with the involvement of heat-activated ion channels such as transient receptor potential (TRP) channels in VGNs and RGNs as shown by Albert et al. [32]. Selective blockade of the laser evoked voltage variations by micromolar concentrations of ruthenium red and RN1734 have identified thermosensitive TRPV4 channels as the primary effectors of the chain reaction triggered by $1,875 \mathrm{~nm}$ laser pulses. It is also consistent with the work on HEK293 cells transfected with TRPV 1, 2, and 3 by Yao et al. [21], showing that specific thermal TRP ion channels were activated at specific temperatures. However, in vivo neural activations of frog and rat sciatic nerves have shown that a thermal gradient more than a threshold temperature is required to initiate APs [13]. Nerves at temperature of 0 and $25^{\circ} \mathrm{C}$ were stimulated at the same radiant exposure thresholds. This suggests that the mechanisms supporting the biological responses to the IR irradiation may differ depending on the cell type. Additional investigations at different room temperatures (for instance $37^{\circ} \mathrm{C}$ ) could confirm that a temperature threshold is required to elicit APs on VGNs and RGNs.

If it has been reported that cells die at temperatures of about $70^{\circ} \mathrm{C}$ in a few seconds [34], Simanovskii et al. [35] have shown that the temperature threshold leading to cellular death rises with decreasing pulse duration, and for pulses of 10 milliseconds it reaches $80^{\circ} \mathrm{C}$. In addition, no cell damages were observed with temperature jumps over $59^{\circ} \mathrm{C}$ with a 100 milliseconds pulse duration on HEK293 cells [21]. Finally, on the assumption of using the heat induced by the laser absorption on the retina as an activation mechanism of the ganglion cell layer, Heussner et al. [36] have presented the concept of a durable excitation for prosthesis applications.

\section{CONCLUSION}

In summary, we have demonstrated that infrared laser radiations provide a possible way for safely stimulating RGNs and VGNs. Stimulation thresholds clearly depend on the water optical absorption coefficient. Local temperature measurements show that a constant temperature rise is required to trigger neurons independently of variable energy thresholds. Finally, such a technique may be valuable for contactless neuronal activity controls on single neurons without genetic or chemical manipulations.

\section{ACKNOWLEDGMENTS}

We are grateful to Dr. Yves Rouillard (Institut d'Electronique du Sud), Guillaume Tatur (ARAMAV) and Dr. H. Boukhaddaoui (Institut des Neurosciences de Montpellier) for technical assistance and to Dr. Guy Laeners and Dr. Christian Hamel (Institut des Neurosciences de Montpellier) for helpful comments.

\section{REFERENCES}

1. Callaway EM, Yuste R. Stimulating neurons with light. Curr Opin Neurobiol 2002;12(5):587-592.

2. Ghezzi D, Menegon A, Pedrocchi A, Valtorta F, Ferrigno G. A micro-electrode array device coupled to a laser-based system for the local stimulation of neurons by optical release of glutamate. J Neurosci Methods 2008;175(1):70-78.

3. Kramer RH, Chambers JJ, Trauner D. Photochemical tools for remote control of ion channels in excitable cells. Nat Chem Biol 2005;1:360-365.

4. Boyden ES, Zhang F, Bamberg E, Nagel G, Deisseroth K. Millisecond-timescale, genetically targeted optical control of neural activity. Nat Neurosci 2005;8:1263-1268.

5. Nikolic K, Grossman N, Grubb M, Burrone J, Toumazou C, Degenaar P. Photocycles of channelrhodopsin-2. Photochem Photobiol 2009;85(1):400-411.

6. Han X, Boyden ES. Multiple-color optical activation, silencing, and desynchronization of neural activity, with singlespike temporal resolution. PLoS ONE 2007;2(3):e299.

7. Banghart M, Borges K, Isacoff E, Trauner D, Kramer RH. Light-activated ion channels for remote control of neuronal firing. Nat Neurosci 2004;7:1381-1386.

8. Chow BY, Han X, Dobry AS, Qian X, Chuong AS, Li M, Henninger MA, Belfort GM, Lin Y, Monahan PE, Boyden ES. High-performance genetically targetable optical neural silencing by light-driven proton pumps. Nature 2010;463: 98-102.

9. Busskamp V, Duebel J, Balya D, Fradot M, Viney TJ, Siegert S, Groner AG, Cabuy A, Forster V, Seeliger M, Biel M, Humphries P, Paques M, Mohand-Said S, Trono D, Deisseroth K, Sahel JA, Picaud S, Roska B. Genetic reactivation of cone photoreceptors restores visual responses in retinitis pigmentosa. Science 2010;329(5990):413-417.

10. Zhang F, Gradinaru V, Adamantidis AR, Durand R, Airan RD, De Lecea L, Deisseroth K. Optogenetic interrogation of neural circuits: Technology for probing mammalian brain structures. Nat Protoc 2010;5:439-456.

11. Hirase H, Nikolenko V, Goldberg JH, Yuste R. Multiphoton stimulation of neurons. J Neurobiol 2002;51(3):237-247.

12. Wells J, Kao C, Jansen ED, Konrad P, Mahadevan-Jansen A. Application of infrared light for in vivo neural stimulation. J Biomed Opt 2005;10(6):1-12.

13. Wells J, Kao C, Konrad P, Milner T, Kim J, MahadevanJansen A, Jansen ED. Biophysical mechanisms of transient optical stimulation of peripheral nerve. Biophys J 2007; 93(7):2567-2580.

14. Izzo AD, Richter CP, Jansen ED, Walsh JY. Laser stimulation of auditory nerve. Lasers Surg Med 2006;38(8):745753.

15. Richter CP, Bayon R, Izzo AD, Otting M, Suh E, Goyal S, Hotaling J, Walsh JT. Optical stimulation of auditory neurons: Effects of acute and chronic deafening. Hear Res 2008;242(1-2):42-51.

16. Izzo AD, Walsh JT, Ralph H, Webb J, Bendett M, Wells J, Richter CP. Laser stimulation of auditory neurons: Effect of shorter pulse duration and penetration depth. Biophys $\mathrm{J}$ 2008;94(8):3159-3166.

17. McCaughey RG, Chlebicki C, Wong BJ. Novel wavelengths for laser nerve stimulation. Lasers Surg Med 2009;42(1): 69-75.

18. Tozburun S, Cilip CM, Lagoda GA, Burnett AL, Fried NM. Continuous-wave infrared optical nerve stimulation for potential diagnostic applications. J Biomed Opt 2010;15(5): 055012 . 
19. Rajguru SM, Richter CP, Matic AI, Holstein GR, Highstein SM, Dittami GM, Rabbitt RD. Infrared photostimulation of the crista ampullaris. J Physiol 2011;589(6):1283-1294.

20. Liang S, Yang F, Zhou C, Wang Y, Li S, Sun CK, Puglisi JL, Bers D, Sun C, Zhenget J. Temperature-dependent activation of neurons by continuous near-infrared laser. Cell Biochem Biophys 2009;53(1):33-42.

21. Yao J, Liu B, Qin F. Rapid temperature jump by infrared diode laser irradiation for patch-clamp studies. Biophys J 2009;96(9):3611-3619.

22. Dittami GM, Rajguru SM, Lasher RA, Hitchcock W, Rabbitt RD. Intracellular calcium transients evoked by pulsed infrared radiation in neonatal cardiomyocytes. J Physiol 2011; 589(6):1295-1306.

23. Shapiro MG, Homma K, Villarreal S, Richter CP, Bezanilla F. Infrared light excites cells by changing their electrical capacitance. Nat Commum 2012;3:736. DOI: 10.1038/ ncomms 1742

24. Niemz M. Laser-tissue interactions: Fundamentals and applications. Berlin: Springer-Verlag; 2002.

25. Freshney IR. Culture of animal cells: A manual of basic technique and specialized applications. New York: John Wiley \& Sons; 2010

26. Valvano JW, Pearce J. Temperature measurements. In: Welch AJ, Van Gemert MJC, editors. Optical-thermal response of laser-irradiated tissue. New York: Plenum Press; 1995. pp 489-534.

27. Greffrath W, Schwarz ST, Büsselberg D, Treede RD. Heatinduced action potential discharges in nociceptive primary sensory neurons of rats. J Neurophysiol 2009;102(1):424436 .
28. Zeeb V, Suzuki M, Ishiwata S. A novel method of thermal activation and temperature measurement in the microscopic region around single living cells. J Neurosci Methods 2004;139(1):69-77.

29. Cesare P, Moriondo A, Vellani V, McNaughton PA. Ion channels gated by heat. Proc Natl Acad Sci 1999;96:76587663 .

30. Kou L, Labrie D, Chylek P. Refractive indices of water and ice in the 0.65 to $2.5 \mu \mathrm{m}$ spectral range. Appl Opt 1993; 32(19):3531-3540.

31. Brugeaud A, Travo C, Demêmes D, Lenoir M, Llorens J, Puel JL, Chabbert C. Control of hair cell excitability by vestibular primary sensory neurons. J Neurosci 2007;27(13): 3503-3511.

32. Albert ES, Bec JM, Desmadryl G, Chekroud K, Travo C, Gaboyard S, Bardin F, Marc I, Dumas M, Lenaers G, Hamel C, Muller A, Chabbert C. TRPV4 channels mediate the infrared laser-evoked response in sensory neurons. J Neurophysiol 2012;107(12):3227-3234.

33. Vogel A, Venugopalan V. Mechanisms of pulsed laser ablation of biological tissues. Chem Rev 2003;103(2):577-644.

34. He X, Bischof JC. The kinetics of thermal injury in human renal carcinoma cells. Ann Biomed Eng 2005;33(4):502510.

35. Simanovskii DM, Mackanos MA Irani AR, O'ConnellRodwell CE, Contag CH, Schwettman HA, Palanker DV. Cellular tolerance to pulsed hyperthermia. Phys Rev E 2006;74(1):11915.

36. Heussner N, Schnichels S, Spitzer M, Bartz-Schmidt KU Stork W. Laser excitation of retinal ganglion cells. Biomed Eng Proc 2011;1:219-222. 